\title{
Clinical Characteristics, Comorbidities, Initial Management and Outcome of COVID-19 Infected Patients Admitted to Intensive Care Unit in Somalia: A National Retrospective Study.
}

Mohamed Farah Yusuf Mohamud ( $\Delta$ m.qadar59@gmail.com )

Mogadishu Somali Turkish Trainin and Research Hospital https://orcid.org/0000-0002-3719-3729

Abdullahi Said Hashi

Mogadishu Somali Turkish Training and Research Hospital

Abdikarim Hussein Mohamed

Mogadishu Somali Turkiosh Training and Research Hospital

Ali Mohamed Yusuf

Mogadishu Somali Turkish Training and Research Hospital

Ibrahim Hussein Ali

Mogadishu Somali Turkish Training and Reseach Hospital

Mohamed Abdi Ahmed

Mogadishu Somali Turkish Training and Research Hospital

\section{Research}

Keywords: Coronavirus disease 2019 (COVID-19), Invasive ventilation, Endotracheal intubation, intensive care unit, Diabetic

Posted Date: September 4th, 2020

DOI: https://doi.org/10.21203/rs.3.rs-66767/v1

License: (c) (1) This work is licensed under a Creative Commons Attribution 4.0 International License. Read Full License 


\section{Abstract}

Purpose: To investigate the clinical characteristics, morbidities, management, and outcomes of COVID-19 Infected patients admitted to the intensive care unit (ICU) in Somalia.

Material and methods: We conducted a retrospective observational study of laboratory 60 confirmed patients with severe acute respiratory syndrome coronavirus 2 (SARS-CoV-2) admitted to an ICU from March 28, to May 28, 2020.The sociodemographic characteristics, comorbidities, exposure history, clinical manifestations (symptoms and signs), laboratory findings, treatment, and outcomes were collected from medical records.

Results: Most of the patients admitted to ICU were men over 59 years of age, and nearly half had diabetes followed by hypertension chronic kidney disease and asthma. The most clinical presentations were dyspnea (91.2\%), Fever (81.1\%), (68.75\%), Fatigue and myalgia (25\%), and Altered level of conscious (16.6\%). Among 48 patients admitted to the ICU, about 24 (50\%) patients had required endotracheal intubation and mechanical ventilation, and 11(29.9\%) patients needed noninvasive ventilation, while 13(27.08\%) patients treated with high-flow oxygen therapy $>15 \mathrm{~L} / \mathrm{min}$ (Table 3). Corticosteroids were administered to most patients $(85.4 \%)$, while $77.1 \%$ of the patients received inhaled bronchodilators and morethan half of the patients administered antibiotics. $58.3 \%$ of the patients had received Oseltamivir, while $22.9 \%$ received Vasopressors.

\section{Conclusion}

This study represents the first description of critically ill patients infected with SARS-CoV-2 admitted to ICU in Somalia. The study identified that elder age, male gender, and diabetic and hypertensive comorbidities as independent risk factors of poor outcomes for patients admitted to the ICU $(p<0.005)$.

\section{Introduction:}

Coronavirus Disease-2019 (COVID-19) had discovered in Wuhan, China, in December 2019 and described as acute viral pneumonia that recently found in humans (1). On 11 March 2020, due to the continually increasing number of COVID-19 cases outside of China, the World Health Organization (WHO) declared as outbreak pandemic(2). On admission, $20-51 \%$ of patients had reported as having at least one comorbidity, with diabetes (10-20\%), hypertension (10-15\%), and other vascular diseases (including the cardiac and brain) $(7-40 \%)$ being most common $(1,3)$. Systemic inflammation, coagulation activation, endothelial dysfunction, Renal failure, myocardial injury, and multiorgan failure had described as the most severe complications of severe COVID-19 $(2,4,5,6)$. Previous studies suggest that $5-20 \%$ of patients with SARS-CoV-2 develop a critical illness that is characterized primarily by acute respiratory distress syndrome $(7,8)$. China, among the COVID-19 infected, $5-32 \%$ had required ICU care $(1,9)$. More than 3 million people worldwide have become infected with Severe acute respiratory syndrome coronavirus 2 (SARS-CoV-2), resulting in more than 215000 deaths, with geographical mortality rates ranging from less than $1-12 \%$ (10). Primary studies from China, Italy, and the United state-reported 
overall mortality ranging from $23-62 \%$ in critically ill patients with COVID-19 $(8,11,12,13)$. By 17 April 2020, 116 confirmed cases were reported in Somalia with $2(1.7 \%)$ recovered cases and $5(4.3 \%)$ deaths and Till now, the reported cases were mostly among adults, with no pediatric cases reported (14).

In this study, we investigated the clinical characteristics, morbidities, management, and outcomes of COVID-19 Infected patients admitted to the intensive care unit (ICU) in Somalia.

\section{Material And Methods:}

We conducted a retrospective observational study of laboratory 60 confirmed patients with severe acute respiratory syndrome coronavirus 2 (SARS-CoV-2) admitted to an ICU in the De Martino Public Hospital from March 28, to May 28, 2020, with outcomes observed until Jun 30,2020. The study had conducted in a tertiary hospital that has 20 ICU beds. From anticipation of the COVID-19 pandemic in Somalia, this is the only hospital that was nominated as COVID-19 center in Somalia, and critically ill patients with SARSCoV-2 in their catchment areas had transferred to this Hospital. All the medical records of confirmed COVID-19 cases were reviewed at least by two or more health care workers. The sociodemographic characteristics, comorbidities, exposure history, clinical manifestations (symptoms and signs), laboratory and radiological findings, treatment guidelines, and outcomes collected from medical records. All data were entered and analyzed by SPSS version 23. Different researchers performed the double-check of the data to avoid errors. The diagnostic criteria of acute respiratory distress syndrome (ARDS), acute cardiac damage, acute renal impairment, and acute liver injury had based on the corresponding guidelines ( 15 , 16). The criteria for discharge were based on the clinical guidelines for the diagnosis and treatment of novel corona virus infection by the Chinese National Health Commission (Trial Version 5) and were grouped into severe and non-severe COVID-19 (17). According to the protocol of WHO guidance (18), all patients were confirmed by a throat swab sample obtained from the upper respiratory tract and defined as a positive result of real-time reverse transcriptase-polymerase chain reaction (RT-PCR) assay and also following the Centers for Disease Control and Prevention (CDC) guidelines. The study has been reviewed and approved by the Medical Ethical Committees of Mogadishu Somalia Turkey Training and Research Hospital, and De Martino Hospital. In agreement with the declaration of Heliski, institutional review board (IRB) allowed the patient consent waiver to review their medical records in this retrospective study where the participants could not contact, and the was no risk to the participants, confidential and, all personality descriptions had excluded from the datasheet (anonymization).

\section{Results:}

Between April 23, to Jun 28, 2020, 443 patients had been admitted to Di Martino Public Hospital with confirmed COVID-19 pneumonia, of whom 48 admitted to ICU, including five patients who had cardiac arrest immediately after admission. Overall, 48 COVID-19 infected patients were admitted to ICU. Of these patients, 24 (50\%) were severely affected patients and required endotracheal intubation and mechanical ventilation, while24 (50\%) patients were moderately affected by the coronavirus and treated with noninvasive respiratory support or high-flow oxygen therapy $>15 \mathrm{~L} / \mathrm{min}$. 
As table 1 shows, the mean age of COVID-19 patients admitted to the ICU was $48 \pm 16$ years, ranging from 19 to 80 years among 48 patients. A total of 34 (\%) of the patients were males and 14) were females. $14.6 \%$ of the patients had a history of exposure to a positive patient, and $79.2 \%$ of patients were unknown exposure, while $6.2 \%$ of patients had no known history of exposure. Most of the COVID-19 patients $(91.6 \%)$ admitted to the ICU had at least one comorbidity with diabetic $(39.5 \% ; n=19)$ and hypertension $(18.75 \%$; $=9$ )as the most common comorbidities followed by Chronic kidney disease and asthma.

The most clinical presentations were dyspnea (91.2\%), Fever (81.1\%), (68.75\%), Fatigue and myalgia (25\%), and Altered level of conscious (16.6\%). Only four (8.3\%) did not develop dyspnea until 4-9days after the onset of symptoms associated with SARS-CoV-2 pneumonia, while 9 (18.75\%) patients did not experience Fever till 4-9days next to the onset COVID-19 related symptom (Table 2).

On admission, the mean white blood cell count(WBC) was $17.6 \mu \mathrm{L}$ and, the mean of hemoglobin (Hb) was $11.9 \mathrm{mg} / \mathrm{dl}$, while the mean platelets were 238.4. Only 12 (\%) patients had Anemia and, seven patients (67\%) had an absolute lymphocyte count of fewer than 1000 cells/ $\mu \mathrm{L}$. Most of the patients had normal renal and liver function tests. Only 9 (18.75\%) patients had abnormal liver function taste, while 3 (6.25\%) patients having SARS-CoV-2 infection those admitted to the ICU had azotemia. Patients with increased CRP were more than those within the normal range (Table 2).

Among 48 patients those admitted to the ICU, about 24 (50\%) patients had required endotracheal intubation and mechanical ventilation, and 11(29.9\%) patients needed noninvasive ventilation, while 13(27.08\%) patients treated with high-flow oxygen therapy > $15 \mathrm{~L} / \mathrm{min}$ (Table 3 ). More than two-thirds of patients admitted to the ICU those required endotracheal intubation and mechanical ventilation were those elder than 65 years and those having at least one comorbid disease.

Corticosteroids were administered to most patients (85.4\%), while $77.1 \%$ of the patients received inhaled bronchodilators and Morethan half of the patients administered antibiotics. $58.3 \%$ of the patients had received Oseltamivir, and $22.9 \%$ received Vasopressors.

Nearly two-thirds of patients died in the intensive care unit, while 14.5\% were still in ICU required for further management. After successful management, $22.9 \%$ of the patients transferred to the non-ICU inpatient and only one lucky patients discharged to the home on foot.

\section{Discussion:}

This study represents the first and most comprehensive study of patients admitted to ICU with COVID-19related critical illness reported to date in Somalia. COVID-19 is a pandemic disease that hit hard throughout the globe, yet no known treatment or vaccination available at the time.

This study included a total of 48 patients admitted in the intensive care unit with SARS-CoV-2 infection in Di Martino Hospital that is the only hospital nominated as COVID-19 center in Somalia between April 
Most of the patients admitted to ICU were men over 59 years of age, and nearly half had diabetes.

In the present study, the majority of patients admitted to the ICU because of acute hypoxemic respiratory failure that required respiratory support.

We identified elder that age, male gender, and diabetic and hypertensive comorbidities as independent risk factors of poor outcomes for patients admitted to the ICU $(p<0.005)$.

About $10.8 \%$ of the total patients admitted to the hospital due to COVID-19 were admitted to the ICU. Half of the patients admitted to the ICU had required endotracheal intubation and invasive mechanical ventilation. Of these patients who underwent mechanical ventilation, $12(25 \%)$ patients died, 6(12.5\%) patients remain in ICU, $5(10.4 \%)$ patients transferred to non-ICU inpatients and, only $1(2.08 \%)$ patient was discharged to home by foot. Noninvasive mechanical ventilation had initiated in $11(22.9 \%)$ patients, while $13(27.08 \%)$ patients had required high-flow oxygen therapy $>15 \mathrm{~L} / \mathrm{min}$.

Similarly to the present study, the use of noninvasive ventilation reported as $19 \%$ in Washington State, US, $42 \%$ in Wuhan, and $62 \%$ in Wuhan, China (note, this value included patients receiving high-flow nasal cannula) $(20,19,1)$.

In the present study, the number of patients required invasive mechanical ventilation higher than that recently reported for other ICU patients: $42 \%$ (Wuhan, China) and $47 \%$ (Wuhan, China) $(8,19)$, while another study in Washington State had reported about $71 \%$ of patients admitted to ICU had required invasive mechanical ventilation that is much more than when compared to our study (20).

In our study, among half of the patients admitted to the intensive care unit died due to acute respiratory distress syndrome (ARDS) and other COVID-19 complications. This higher rate of death was related to delayed seek for the medical care of the patients due to some cultural issues, short coverage of proper and adequate intensive care units, and lack of experience with the use of mechanical ventilators and other invasive facilities in our country. Similar studies in Wuhan, Lombardy, and Seattle had shown a higher mortality rate between $50-80 \%$ for critically ill COVID-19 infected patients admitted to the ICU (8, $11,12)$.

\section{Conclusion:}

This study represents the first description of critically ill patients infected with SARS-CoV-2 admitted to ICU in Somalia. The study identified that elder age, male gender, and diabetic and hypertensive comorbidities as independent risk factors of poor outcomes for patients admitted to the ICU $(p<0.005)$.

\section{Declarations}

Ethics approval and consent to participate: 
We obtained an approvalletter from the review board of Mogadishu Somali Turkish Training and Research Hospital and written informed consent was waived (MSTH/3712) because the study was retrospective and collected from medical records. We declare that we have followed the protocols of our work center. Patient data confidentiality was respected.

\section{Availability of data and materials:}

The data that support the findings of this study are available from Mogadishu Somali Turkish Training and Research Hospital. Data are however available from the authors upon reasonable request and with permission of Mogadishu Somali Turkish Training and Research Hospital.

\section{Competing of interes and Funding:}

We declare that we have no competing interests and funding source.

\section{Authors' contributions}

M.F.Y.M. and A.S.H:. Writing of the manuscript, final revision of data, statistical tables, and and finalization of the research manuscript. I.H.A and A.M.Y.: idea of the research, supervision, and revision of data. A.H.M and M.A.A.: collecting data, and helping in writing of the manuscript. All authors read and approved the final manuscript.

\section{Acknowledgements:}

Not applicable.

\section{Consent For Publication:}

Not applicable.

\section{References}

1. Huang C, Wang Y, Li X, Ren L, Zhao J, Hu Y, Zhang L, Fan G, Xu J, Gu X, Cheng Z. Clinical features of patients infected with 2019 novel coronavirus in Wuhan, China. The Lancet. 2020 Feb 15; 395(10223):497-506.

2. WHO Director-General'sopeningremarks at themediabriefing on COVID-19: 11 March 2020. PublishedMarch 11, 2020. AccessedMarch 30, 2020. https://www.who.int/dg/speeches/detail/ whodirector-general-s-opening-remarks-at-themedia-briefing-on-covid-19-11-march-2020.

3. Liu K, Fang YY, Deng Y, Liu W, Wang MF, Ma JP, Xiao W, Wang YN, Zhong MH, Li CH, Li GC. Clinical characteristics of novel coronavirus cases in tertiary hospitals in Hubei Province. Chinese medical journal. 2020 Feb 7.

4. Zhou F, Yu T, Du R, Fan G, Liu Y, Liu Z, Xiang J, Wang Y, Song B, Gu X, Guan L. Clinical course and risk factors for mortality of adult inpatients with COVID-19 in Wuhan, China: a retrospective cohort study. 
The lancet. 2020 Mar 11.

5. Ruan Q, Yang K, Wang W, Jiang L, Song J. Clinical predictors of mortality due to COVID-19 based on an analysis of data of 150 patients from Wuhan, China. Intensive care medicine. 2020 May;46(5):846-8.

6. Tang N, Bai H, Chen X, Gong J, Li D, Sun Z. Anticoagulant treatment is associated with decreased mortality in severe coronavirus disease 2019 patients with coagulopathy. Journal of thrombosis and haemostasis. 2020 May;18(5):1094-9.

7. Wu Z, McGoogan JM. Characteristics of and important lessons from the coronavirus disease 2019 (COVID-19) outbreak in China: summary of a report of 72314 cases from the Chinese Center for Disease Control and Prevention. Jama. 2020 Apr 7;323(13):1239-42.

8. Yang X, Yu Y, Xu J, Shu H, Liu H, Wu Y, Zhang L, Yu Z, Fang M, Yu T, Wang Y. Clinical course and outcomes of critically ill patients with SARS-CoV-2 pneumonia in Wuhan, China: a single-centered, retrospective, observational study. The Lancet Respiratory Medicine. 2020 Feb 24.

9. Guan WJ, Ni ZY, Hu Y, Liang WH, Ou CQ, He JX, Liu L, Shan H, Lei CL, Hui DS, Du B. Clinical characteristics of coronavirus disease 2019 in China. New England journal of medicine. 2020 Apr 30;382(18):1708-20.

10. Chen N, Zhou M, Dong X, et al. Epidemiological and clinical characteristics of 99 cases of 2019 novel coronavirus pneumonia in Wuhan, China: a descriptive study. Lancet. 2020;395(10223):507-513.

11. Grasselli G, Zangrillo A, Zanella A, Antonelli M, Cabrini L, Castelli A. Baseline Characteristics and Outcomes of 1591 Patients Infected With SARS-CoV-2 Admitted to ICUs of the Lombardy Region, Italy. Jama [Internet]. 2020; 1-8.

12. Bhatraju PK, Ghassemieh BJ, Nichols M, Kim R, Jerome KR, Nalla AK, Greninger AL, Pipavath $S$, Wurfel MM, Evans L, Kritek PA. Covid-19 in critically ill patients in the Seattle region-case series. New England Journal of Medicine. 2020 May 21;382(21):2012-22.

13. Richardson S, Hirsch JS, Narasimhan M, Crawford JM, McGinn T, Davidson KW, Barnaby DP, Becker LB, Chelico JD, Cohen SL, Cookingham J. Presenting characteristics, comorbidities, and outcomes among 5700 patients hospitalized with COVID-19 in the New York City area. 2020 Apr 22.

14. Mohamud, M. F. Y., Mohamed, Y. G., Ali, A. M., \& Adam, B. A. (2020). Loss of Taste and Smell are Common Clinical Characteristics of Patients with COVID-19 in Somalia: A Retrospective Double Centre Study. Infection and Drug Resistance, 13, 2631.

15. Rubenfeld GD, Thompson T, Ferguson ND, Caldwell E, Fan E, Camporota L, Slutsky AS. Acute respiratory distress syndrome. The Berlin definition. JAMA. 2012;307(23):2526-33.

16. Gao C, Wang Y, Gu X, Shen X, Zhou D, Zhou S, Huang JA, Cao B, Guo Q. Association between cardiac injury and mortality in hospitalized patients infected with avian influenza A (H7N9) virus. Critical care medicine. 2020 Apr;48(4):451.

17. National Health Commission. Guidelines for the Diagnosis and Treatment of Novel Coronavirus (2019-nCoV) Infection by the National Health Commission (Trial Version5). 
18. Clinical management of severe acute respiratory infection when novel coronavirus(nCoV) infection is suspected: interim guidance, 25 January 2020. Published January 25, 2020.Accessed March 30 , 2020.https://apps.who.int/iris/handle/10665/330854.

19. Wang D, Hu B, Hu C, Zhu F, Liu X, Zhang J, Wang B, Xiang H, Cheng Z, Xiong Y, Zhao Y. Clinical characteristics of 138 hospitalized patients with 2019 novel coronavirus-infected pneumonia in Wuhan, China. Jama. 2020 Mar 17;323(11):1061-9.

20. Arentz M, Yim E, Klaff L, Lokhandwala S, Riedo FX, Chong M, Lee M. Characteristics and outcomes of 21 critically ill patients with COVID-19 in Washington State. Jama. 2020 Apr 28;323(16):1612-4. 